\title{
Tonal Noise Prediction of a Distributed Propulsion Unmanned Aerial Vehicle
}

\author{
Kyle A. Pascioni* \\ National Institute of Aerospace, Hampton, VA 23666 \\ Stephen A. Rizzi ${ }^{\dagger}$ \\ NASA Langley Research Center, Hampton, VA 23681
}

\begin{abstract}
Noise is expected to be a major barrier of unmanned aerial vehicles (UAVs) to public acceptance. A noise prediction scheme is introduced in this paper and applied to a specific vehicle configuration, namely, the Greased Lightning-10. Results herein will be used to demonstrate the feasibility of incorporating a noise constraint within the flight control system of a distributed electric propulsion vehicle by modifying commands for lownoise operation over sensitive areas, e.g., communities, schools, etc. Steady loading and thickness noise signatures of single propellers are computed using the Propeller Analysis System of the NASA Aircraft NOise Prediction Program. The individual signatures are then superposed at far field observers after applying corrections for spherical spreading and phase delays based on straight ray propagation. Two-propeller source fields are verified using analytical directivity patterns of monopoles. Notional effects of rotation rate, rotation direction, and relative propeller phase are given. Under ideal circumstances and equivalent RPM, random phasing, which occurs in most small UAVs, can produce up to 20 $\mathrm{dB}$ uncertainty in the tonal sound pressure level at a given ground observer. Additionally, directivity modification via relative propeller phase control is shown to have great potential as a noise reduction technique. This paper will focus on the forward flight mode but will also briefly discuss the vertical flight mode.
\end{abstract}

\section{Nomenclature}

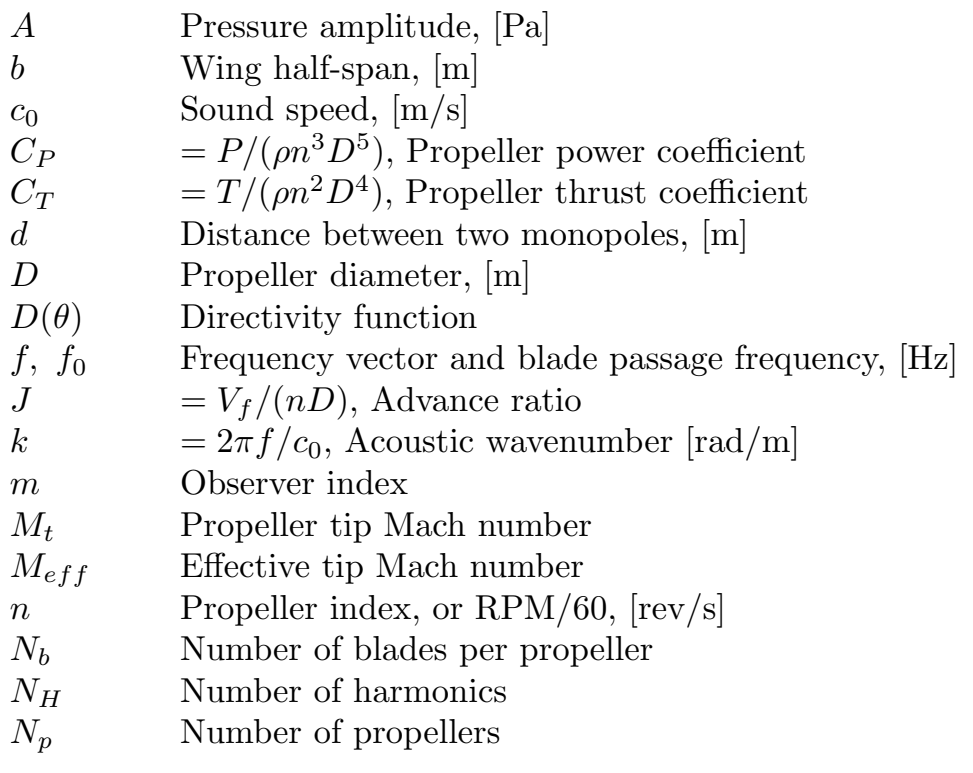

${ }^{*}$ Research Engineer, Member AIAA

${ }^{\dagger}$ Senior Researcher for Aeroacoustics, Aeroacoustics Branch, Fellow AIAA 
$O A S P L \quad$ Overall sound pressure level, [dB re. $20 \mu \mathrm{Pa}$ ]

$P \quad$ Propeller power, $[\mathrm{W}]$

$P W L \quad$ Sound power level, [dB re. $10^{-12}$ Watts]

$r_{m, n} \quad$ Distance between observer $m$ and propeller $n,[\mathrm{~m}]$

$R_{s} \quad$ Radius of source hemisphere, [m]

$S P L \quad$ Sound pressure level, [dB re. $20 \mu \mathrm{Pa}]$

$t \quad$ Time vector, $[\mathrm{s}]$

$T \quad$ Propeller thrust, $[\mathrm{N}]$

$V_{f} \quad$ Vehicle flight speed, $[\mathrm{m} / \mathrm{s}]$

$\mathbf{x}=\left(x_{1}, x_{2}, x_{3}\right)$, Observer coordinates, $[\mathrm{m}]$

$\mathbf{y}=\left(y_{1}, y_{2}, y_{3}\right)$, Propeller coordinates, $[\mathrm{m}]$

$\alpha \quad$ Propeller angle of attack, [deg.]

$\eta \quad=C_{T} J / C_{P}$, Propeller efficiency coefficient

$\Omega \quad$ Propeller rotation rate, $[\mathrm{rad} / \mathrm{s}]$

$\rho \quad$ Ambient density, $\left[\mathrm{kg} / \mathrm{m}^{3}\right]$

$\psi, \psi_{r} \quad$ Propeller azimuthal angle and relative phase, [deg.]

$\psi_{\text {mono }} \quad$ Relative phase between two monopoles

$\theta \quad$ Azimuthal angle, [deg.]

\section{Introduction}

ISTRIBUTED electric propulsion (DEP) systems are becoming increasingly popular because of their ability to distribute propulsors in many locations on the vehicle, not just near the power source. For aircraft designers, DEP opens up new degrees of freedom within aerodynamics, vehicle control, and acoustics, to name a few. New small/medium unmanned aerial vehicles (UAVs), urban air mobility (UAM) systems, and thin/short haul aircraft concepts and demonstrators exploit DEP for different purposes. Noise is anticipated to be one of the key barriers to entry into service for these platforms.

DEP aircraft noise is expected to be very different in comparison to conventional aircraft. ${ }^{1}$ Coherent sources, e.g., propellers operating at the same RPM, can generate complicated directivity patterns that are difficult to predict, particularly when progressing from ideal conditions to realistic flight. While propellers operating at different rotation rates eliminate the phase cancellation process, slightly different rates can produce time signatures with time-varying characteristics. Therefore, a noise prediction tool should not only encompass overall levels but also have the ability to accurately reproduce acoustic time histories for auralization and human perception tests. Given the importance of the waveform, traditional A-weighting of the sound pressure spectrum may not be an appropriate metric to fully reflect human response. ${ }^{2}$ To this end, the resulting noise model herein will be a time domain formulation. At this point, however, the most appropriate acoustic metrics for these vehicles are not yet established. As a placeholder, mean-square pressures of the time series will be used to quantify trends.

Generally speaking, noise reduction techniques typically focus on noise or flow control directed at the source. While this pathway obviously shows its merit, the current work is part of a project that focuses on modifying flight operations, e.g., reduce the flight speed or modify the path as a means of noise reduction. To enable strategic decision making by the controller, a noise prediction tool is needed. Results of this paper are included in Galles et al., ${ }^{3}$ and serve as the basis for a surrogate noise model that is coupled with the flight control system. There, the feasibility of incorporating a noise metric into the control system of a UAV is demonstrated. Automatic management of the commanded flight speed during a mission has the potential to ensure a predefined noise target is not exceeded. For example, the controller can be programmed to minimize time-to-destination without exceeding noise constraints when flying over noise-sensitive areas (e.g., communities, schools, etc.).

The demonstration vehicle selected is the Greased Lightning-10 (GL-10). ${ }^{4}$ This selection was made on the basis of its modes of operation and on the availability of a flight control simulation. The GL-10 has a Vertical Takeoff and Landing (VTOL) mode via wing- and tail-tilt allowing the propulsion system to act as a set of rotors. In forward flight mode, the propeller shaft angle is aligned (or slightly offset by the propeller angle of attack) with the flight direction similar to conventional propeller-driven aircraft.

The acoustic model will focus on tonal propeller noise generated by blade motion and steady blade 
loading. Broadband sources due to airfoil self-noise, ${ }^{5}$ or noise due to unsteady loading via nonuniform inflow or turbulence ingestion are neglected at this time. It should be noted that such sources are expected to become more relevant for smaller-scale propellers with low tip Mach numbers. ${ }^{6}$ Electric motor noise, ${ }^{7}$ airframe noise, and propeller-propeller or propeller-airframe interactions are also not modeled herein.

This paper begins by introducing the chosen vehicle configuration and its propeller flight envelope. A brief discussion on how the results will be implemented into the control strategy follows. Additional details can be found in Galles et al. ${ }^{3}$ Then, single propeller tonal noise predictions are presented, along with their superposition to simulate the effect of DEP on a far field observer. Verification of the superposition is also given. Mach number scaling with RPM and notional effects of other parameters such as rotation direction and both fixed and random relative blade azimuthal positions (which will be referred to as propeller phase) are given. The potential of directivity modification via phase control is examined via two examples. Finally, the vertical flight mode is briefly discussed.

\section{GL-10 Vehicle Configuration}

The GL-10 is a $50 \%$ scale vehicle concept developed by NASA to combine long endurance with VTOL technology. ${ }^{8}$ Figure 1a shows the GL-10 in hover mode. The DEP system includes eight propellers along the wing and two propellers along the tail. A wing- and tail-tilt command allows the propulsion system to act as a set of rotors during takeoff and landing operations. Midflight transition occurs to enter a conventional operating state during forward flight. Each propeller is composed of three AeroNaut 16x8 CAM folding carbon blades with a diameter of $0.4064 \mathrm{~m}$.

Figure $1 \mathrm{~b}$ is a schematic of the propeller locations relative to the vehicle's roll axis. The dimensions are written as a fraction of the wing half-span, $b=1.6 \mathrm{~m}$, with propellers labeled $n_{1}-n_{10}$. Notice that the propellers are mounted on a swept wing with angle of $10.6^{\circ}$.

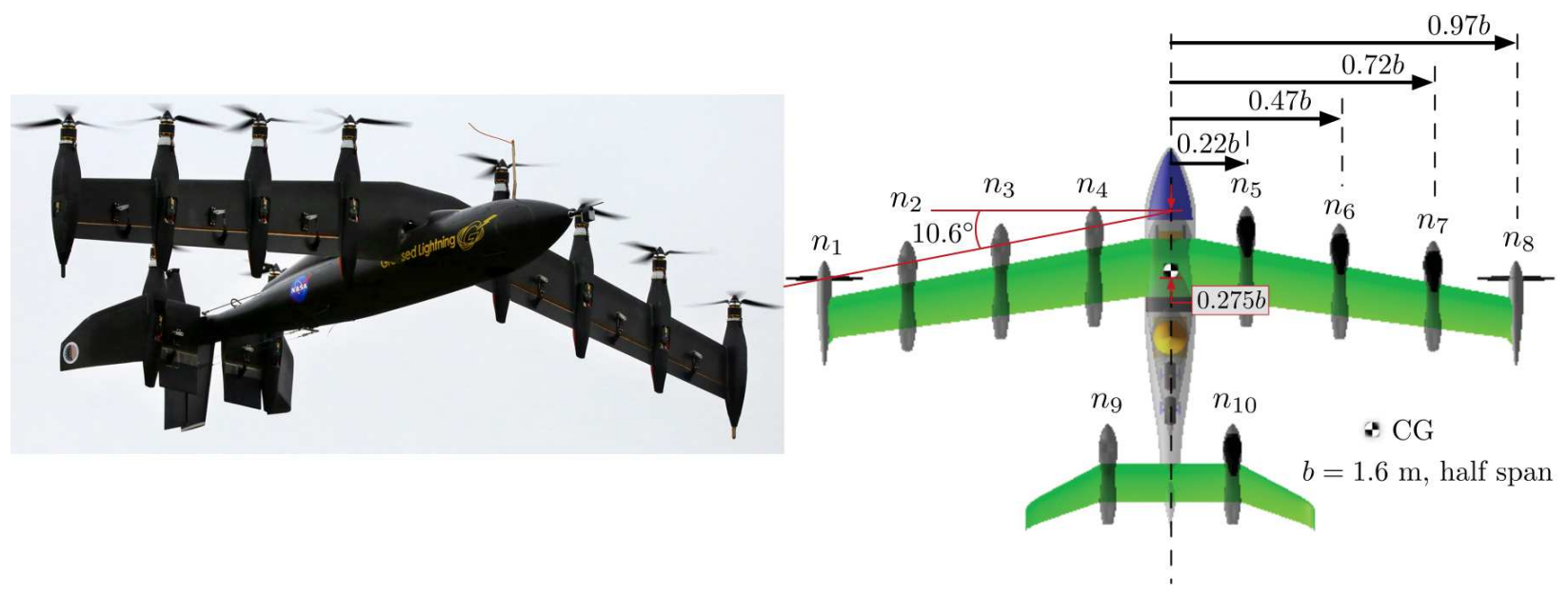

(a)

(b)

Figure 1. GL-10 in (a) hover mode and (b) top view of the forward flight configuration. Propellers are labeled $n_{1}-n_{10}$ in which $n_{9}=(-0.183 b,-0.417 b), n_{10}=(0.183 b,-0.417 b)$ relative to the CG.

\section{II.A. Flight Envelope}

VTOL, transition, and forward flight are the three operating modes that encompass a single GL-10 mission. The work herein focuses on the forward flight configuration in which the propulsion system acts like a set of propellers (the vertical flight mode is only discussed in section V). This vehicle may utilize only the two outboard wing propellers driven by a hybrid diesel electric motor during forward flight, ${ }^{8}$ while the remaining wing propellers deploy only during transition and hover. However, the noise prediction in the following sections will focus on the current configuration in which all ten propellers are in use.

Propeller inflow angle, hereafter referred to as the angle of attack, is restricted to $\alpha= \pm 8^{\circ}$ for the noise analysis. Typical cruise speeds range from $20-30 \mathrm{~m} / \mathrm{s}$. The total thrust requirement sets the RPM of all propellers, with a maximum rotation rate defined by the propeller manufacturer of 9,000 RPM. It is likely 
that off-design propeller operation may benefit acoustic performance. The advance ratio, $J=V_{f} /(n D)$, is a nondimensional parameter used to bound the range of rotation rates. In the definition, $V_{f}$ is the vehicle flight speed, $n$ is the rotation rate in revolutions per second, and $D$ is the propeller diameter. The minimum rotation rate is not constant but rather has dependence on flight speed to avoid the windmilling effect, ${ }^{9}$ in which a set rotation rate is lower than a freely rotating propeller, e.g., without torque supplied by the motor. Under this circumstance, work is being done by the fluid (negative thrust) rather than the propeller performing work on the fluid (positive thrust). Figure 2(a) gives the vehicle flight speed and RPM combinations that generate positive thrust. Additionally, the thrust, power, and efficiency coefficients are given as a function of advance ratio in Fig. 2(b) for a single propeller with uniform inflow. These performance characteristics are estimated using the PAS-ANOPP codeset which will be discussed in section III.A.

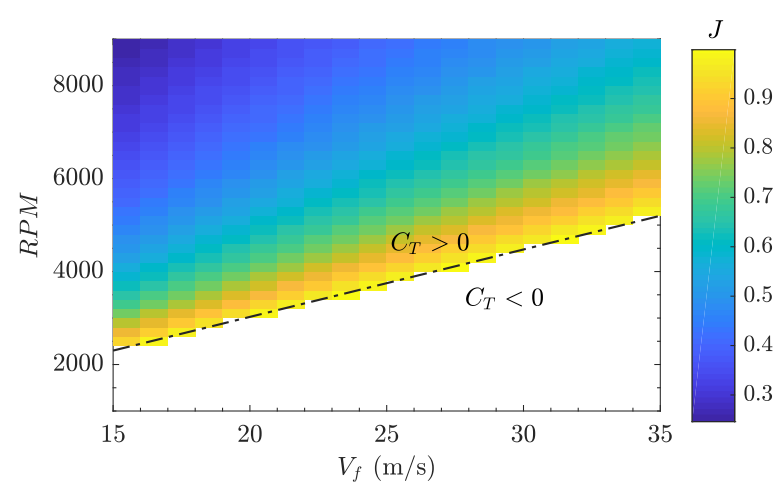

(a)

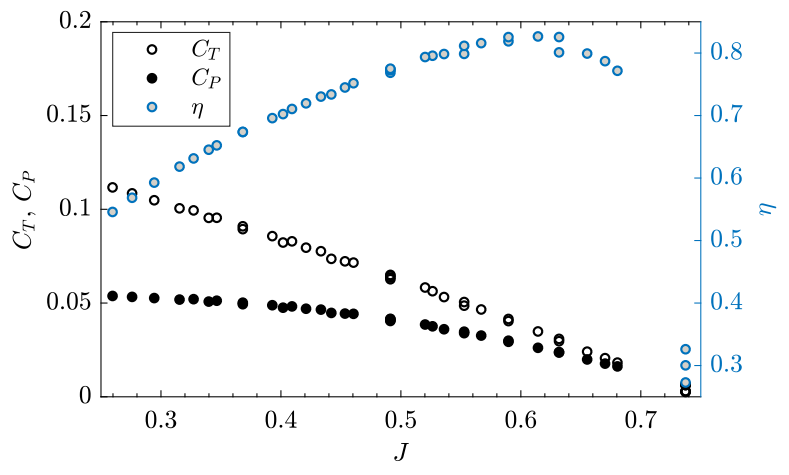

(b)

Figure 2. Parameters describing the propeller operating regime determined by the flight envelope, where (a) gives the advance ratio for various flight speed and RPM combinations, and (b) performance coefficients estimated by PAS.

\section{II.B. Flight Controller}

Control authority is based on several modules summarized in the block diagram of Fig. $3 .{ }^{3}$ A Path Following Controller creates appropriate roll, pitch, and yaw commands to maintain a given flight profile. An allocation matrix passes commands to each propeller and control surface after the Dynamics Controller supervises aircraft stability requirements. No actual flight tests are used for this work. Rather, a Vehicle Dynamics Model simulates flight for this feasibility study and has been calibrated via wind tunnel tests. ${ }^{8}$

During flight, sensor data of individual operating propeller RPM and control surface positions are collected to provide feedback for the control system. The current work, along with Galles et al., ${ }^{3}$ proposes an additional set of control laws to work in series with the existing platform, viz., the Acoustics Controller. A noise metric, chosen to be total radiated sound power, is derived from the vehicle operating state in the Acoustic Model using the methods described in the following sections. Total sound power is selected given its simplicity, although more informative quantities with spatial dependence and weighted based on human perception are currently being considered. Acoustic targets are set for operation that could be imposed when flying over noise sensitive areas. Conversely, a higher acoustic target can be set for flight over commercial areas in which noise restrictions are relaxed. The high-noise target is an option in an effort to minimize time-to-destination, and if desired, could correspond to the maximum vehicle flight speed. A proportionalintegral-derivative acoustic controller drives the commanded flight speed to minimize the error between the current operating noise level and the predefined acoustic target.

\section{Noise Prediction Methods}

The following sections describe the inner workings of the Acoustic Model in Fig. 3. Single propeller predictions are achieved using a low-fidelity code set. The superposition of single propeller predictions to estimate the total tonal propulsive noise will then be discussed. Various parameters are then independently adjusted to understand their relative impact. 


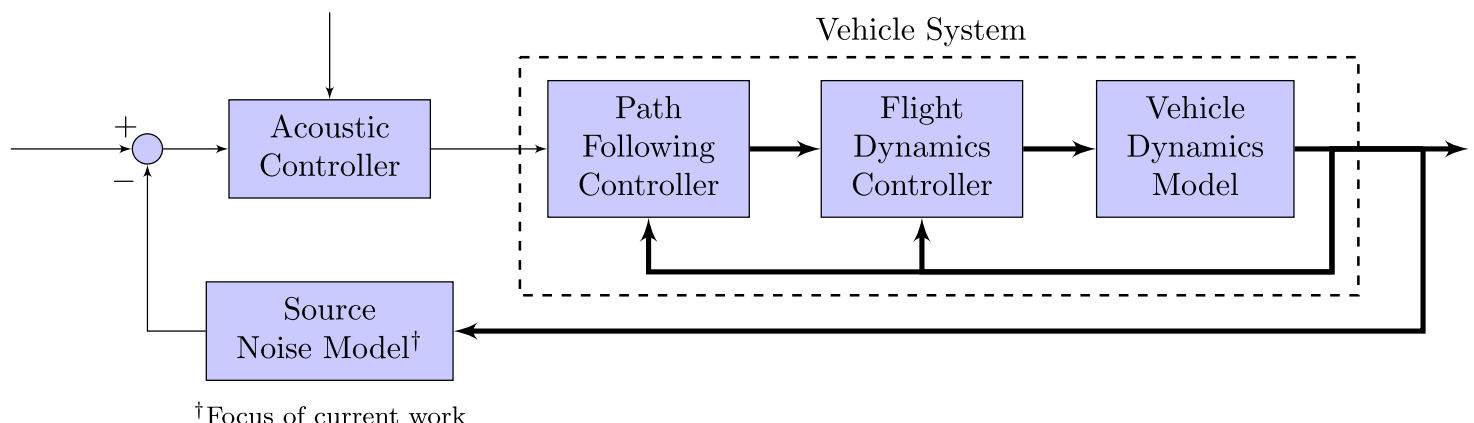

Figure 3. Block diagram of control system for the GL-10 vehicle. This paper is concerned with building an acoustic model to serve as a flight controller informant for low-noise decision making. Figure from Galles et al. ${ }^{3}$

\section{III.A. Single Propeller Prediction}

The Propeller Analysis System ${ }^{10}$ (PAS) module of the Aircraft NOise Prediction Program ${ }^{11}$ (ANOPP) is used to estimate the acoustic signature of a single propeller. To do so, the blade geometry is first discretized into 22 blade sections along its span. Two dimensional potential flow is computed about each Joukowski transformed blade section. The Kutta condition sets the blade element circulation. Inflow velocity components are prescribed by Blade Element Momentum Theory (BEMT) in which radial velocity components are neglected. Boundary layer parameters are also estimated and incorporated into loading (e.g., lift and pressure) and performance coefficients (e.g., power and thrust). The laminar boundary layer region is computed by the method of Young and Squire, ${ }^{12}$ while the turbulent region is prescribed by Truckenbrodt's method. ${ }^{12}$ With the blade loading defined, two types of deterministic tonal noise, loading and thickness noise, are predicted using Farassat's F1A formulation ${ }^{13}$ of the FW-H equation. The complex acoustic pressure spectrum is stored on a "source hemisphere" 12 radii away with 5 degree resolution.

The loading is azimuthally symmetric when the propeller is at a zero degrees angle of attack with a prescribed uniform inflow. Here, the azimuth refers to the angle in the plane of rotation. Conversely, a nonzero angle of attack induces unsteady periodic loading as the propeller completes a revolution. Harmonics of the blade passage frequency are excited and increase in strength relative to the fundamental blade passage frequency. PAS is capable of modeling the asymmetric loading by applying a coordinate transformation to the inflow angle about the azimuth. Experimental measurements display a favorable agreement with PAS predictions for modest nonzero angles of attack. ${ }^{14}$ Noise contours on a ground plane are given in Fig. 4 for various angles. Relative to $\alpha=0^{\circ}$, the overall sound pressure levels increase on the side of increased blade loading while being reduced on the opposing side. While PAS is capable of modeling small changes in angle of attack, the results in the following sections will assume $\alpha=0^{\circ}$ in an effort to reduce the number of parameters being studied.

For small diameter propellers where the tip Mach number is low, additional source mechanisms are likely to play an important role. These include self-noise and noise due to turbulence ingestion (i.e., a nonuniform inflow). However, these physics are neglected at this time. Propeller-propeller interaction noise, propellerairframe interaction noise, and airframe noise are also not incorporated. That said, the current formulation is amenable to including additional sources to increase fidelity and will be sought in future efforts.
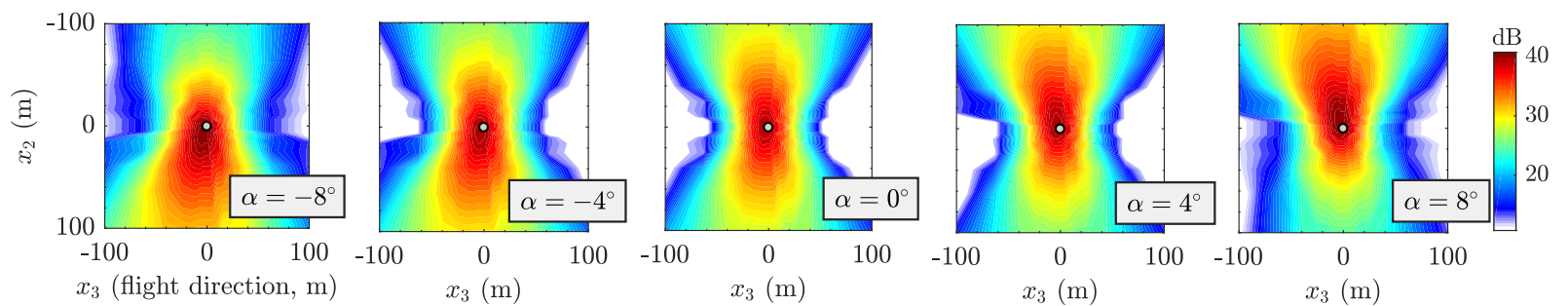

Figure 4. The effect of angle of attack on $O A S P L$ contours on a ground plane produced by a single propeller. Refer to Fig. 5(b) for the coordinate system. Each case has a rotation rate of 5,000 RPM for a flight speed $V_{f}=20 \mathrm{~m} / \mathrm{s}$ at 30 $m$ altitude, with a counterclockwise rotation direction as viewed from behind the propeller. 


\section{III.B. Distributed Propulsion}

Several processing steps are performed to determine the effects of a multipropeller system. An instance of the complex acoustic pressure hemisphere obtained from PAS, i.e., a source hemisphere, is placed at each GL-10 propeller location as defined in Fig. 1b. A ground plane with a set of observer locations $\mathbf{x}=\left(x_{1}, x_{2}, x_{3}\right)$ is centered on the vehicle center of gravity (CG) and moves with the aircraft. Hence, there is no Doppler shift embedded in these simulations. The vertical distance from the ground plane to the CG coincides with a cruise altitude of $30.5 \mathrm{~m}$. Atmospheric absorption is not modeled as it is assumed to be a small effect at the frequencies and length scales investigated here. Straight rays from each propeller to each ground observer map out the propagation paths. Intersection of these rays with the source hemispheres determines the query points where the complex acoustic pressure is interpolated. Figure 5(a) illustrates this process. Note that the magnitude and phase are individually linearly interpolated along the source hemisphere surface. The process is repeated for each propeller.

The interpolated pressures are then phase delayed given the distance between the source hemispheres to the ground plane. To clarify, let $R_{s}$ be the radius of a source hemisphere and let the complex acoustic pressure at the interpolation point of Fig. 5 (a) have pressure amplitude $\tilde{A}$ with phase $\tilde{\psi}$. We will use the notation $(\tilde{)})$ to denote an interpolated value. Then, the acoustic phase with frequency $f$ at observer $m$ due to propeller $n$ is $\tilde{\psi}_{m, n}+k\left(r_{m, n}-R_{s}\right)$, in which $k=2 \pi f / c_{0}$ and $c_{0}$ is the sound speed. The distance between source and observer $r_{m, n}=\left|\mathbf{x}_{\mathbf{m}}-\mathbf{y}_{\mathbf{n}}\right|$ as illustrated in Fig. 5(c). Spherical spreading is accounted for by scaling the pressure amplitude based on the additional distance, i.e., $p_{m} / \tilde{A}_{m} \propto R_{s} / r_{m}$. Finally, the contributions of each propeller can be linearly summed at observer $m$,

$$
p_{m}(t)=\sum_{n=1}^{N_{p}} \sum_{n_{h}=1}^{N_{H}} \frac{R_{s}}{r_{m, n}} \tilde{A}_{m, n_{h}, n} \sin (\Psi),
$$

in which

$$
\Psi=2 \pi n_{h} f_{0, n} t+\tilde{\psi}_{m, n_{h}, n}+k_{n_{h}}\left(r_{m, n}-R_{s}\right)+N_{b} n_{h} \psi_{r_{n}} .
$$

The summation occurs across the number of harmonics, $N_{H}$, and the number of propellers, $N_{p}$. Note that PAS can model a propeller rotating in either a counterclockwise or clockwise fashion. Thus, no sign change is needed in equation 1 or 2 to incorporate different rotation directions, as they are already captured in the $(\tilde{)})$ quantities. Five harmonics will be used for the remainder of this work unless noted otherwise. The spectral roll-off is such that higher harmonics $\left(>5 f_{0}, f_{0}\right.$ being the blade passage frequency) are significantly lower in amplitude. Throughout the flight envelope, the sixth harmonic wavers around $-40 \mathrm{~dB}$ relative to the fundamental level. Moreover, retaining higher harmonics would necessitate additional resolution on the source hemispheres for interpolation. The last term of equation $2, \psi_{r, n}$, adjusts the relative angular blade position (or clocking) on the interval $[0,2 \pi)$ of the full azimuth. The number of blades per propeller, $N_{b}$, is used to index the phase based on the blade passage frequency and its harmonics. Time dependence of the final form is preferred over a frequency-domain formulation to permit any noise metric of interest to be calculated.

\section{III.B.1. Verification}

To verify these post-processing steps, we leverage the fact that loading and thickness noise are axisymmetric about $y_{3}$ (see Fig. 5(b)) when $\alpha=0^{\circ}$. Hence, the directivity patterns of two or more propellers can be compared to combinations of simple monopole sources along the axis perpendicular to the flight direction. Blackstock ${ }^{15}$ notes that two monopoles spaced a distance of $d$ have the following directivity pattern,

$$
D(\theta)=\cos \left(\frac{k d}{2} \sin (\theta)-\psi_{\text {mono }}\right),
$$

in which $\psi_{\text {mono }}$ is the relative phase between two monopoles pulsing at the same frequency. Figure 6 compares the interference pattern of equation 3 and two propellers at various spacing when $N_{b} \psi_{r}=0$. The PAS results agree well, particularly at low $k d$. As $k d$ is increased, slight discrepancies arise, which can be attributed to the resolution of the source hemispheres. This effect of resolution was validated by decreasing resolution from the original 5 degrees to 10 and 15 degrees (not shown); these latter cases display more noticeable differences. While the patterns are dependent on the distance $d$, the more important quantity is the compactness ratio, $k d$. For instance, doubling the frequency and halving the separation distance yields identical patterns. 


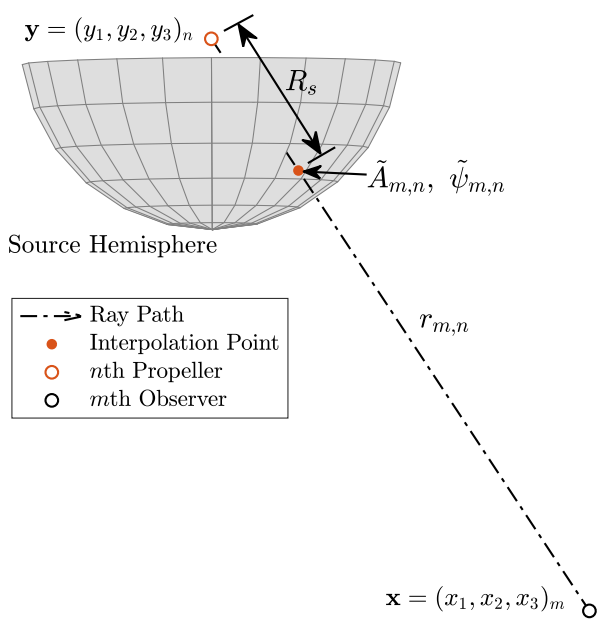

(a)

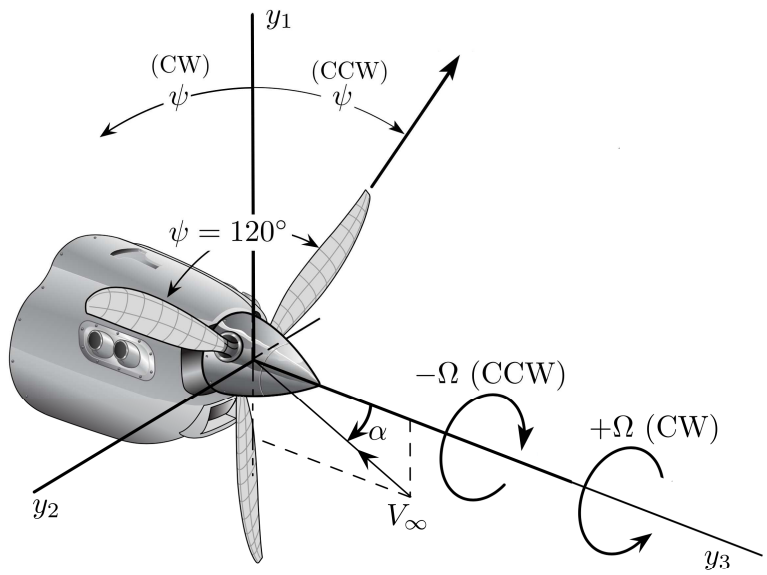

(b)

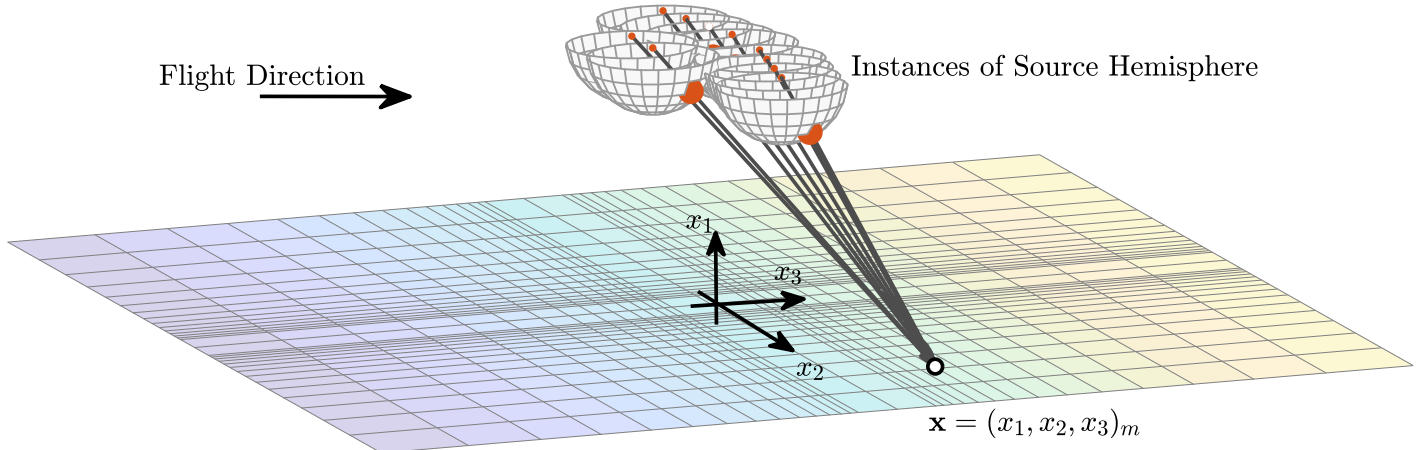

Ground Plane

(c)

Figure 5. Geometric definitions for the (a) source hemisphere in which single propeller PAS acoustic data are obtained and (b) the propeller coordinate system. Note $\psi$ is defined in direction of rotation and spans $360^{\circ}$ over the full azimuth, i.e., each blade is separated by $120^{\circ}$. The ground plane of observers relative to several instances of the source hemisphere is given in (c) to represent the GL-10 DEP configuration (not to scale). 
The relative phase of two sources at the same frequency is also an important parameter, which can alter the directivity pattern. Figure 7 displays how the set of lobes are shifted about the azimuth. Note how complete cancellation occurs at the midline for $\psi_{\text {mono }}=N_{b} \psi_{r}=180^{\circ}$ when the two waveforms are completely out of phase. Again, post-processing a two-propeller system by imposing a relative phase offset, as described in section III.B, shows good agreement with the directivity function.

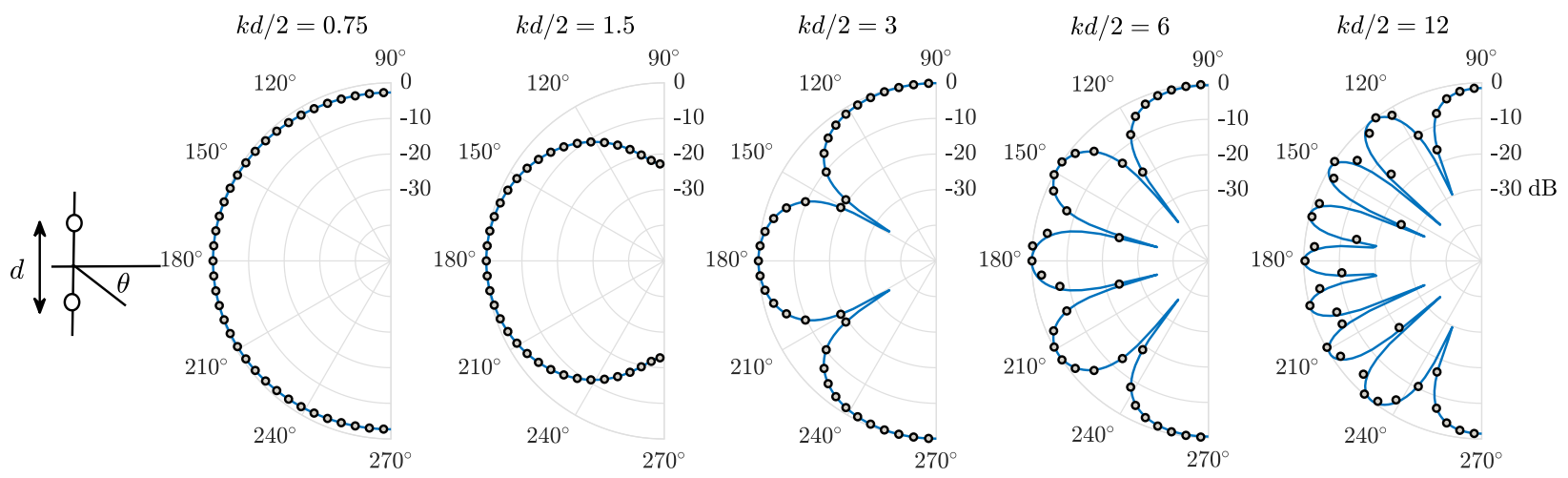

Figure 6. In-plane directivity, $D(\theta)$, of a two-propeller simulation for $\psi_{\text {mono }}=N_{b} \psi_{r}=0^{\circ}$ at various $k d / 2$ values. The solid line is equation 3 and the circles are PAS results at $\alpha=0^{\circ}$, processed using equations 1-2.

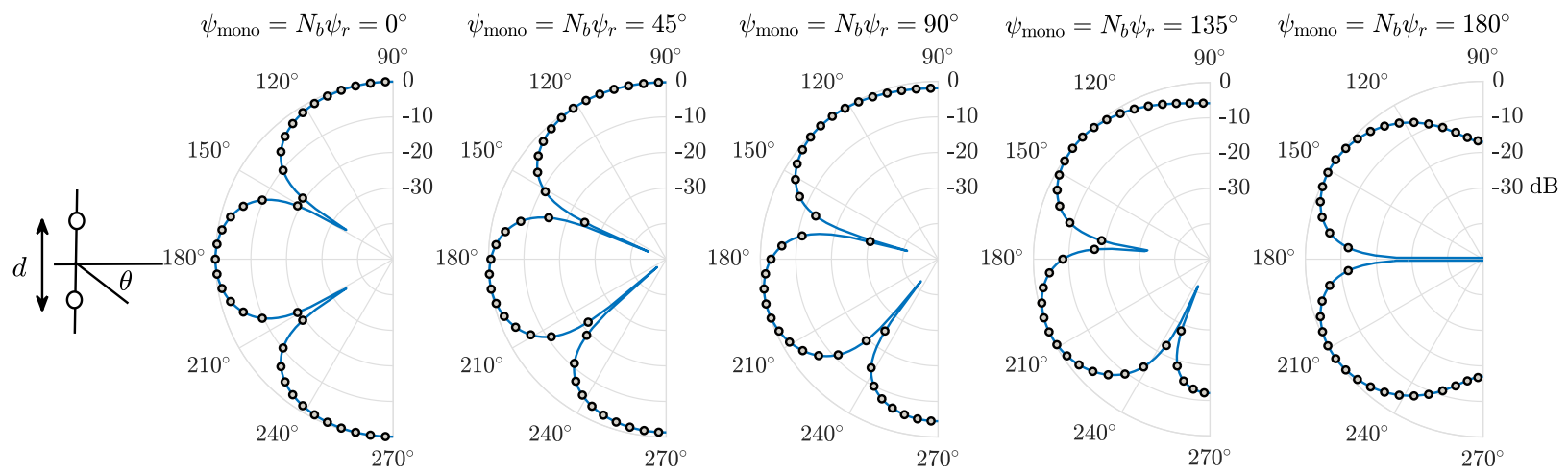

Figure 7. Directivity in the propeller plane, $D(\theta)$, of a two-propeller simulation with various phase offsets for $k d / 2=3$. The solid line is equation 3 and the circles are PAS results at $\alpha=0^{\circ}$, processed using equations 1-2.

\section{Forward Flight Results}

The tonal acoustic signature of the GL-10 is dependent on a number of parameters. Combinations of flight speed, propeller rotation rates, and relative phase offsets will be addressed in this section. The effect of nonaxial inflow (i.e., $\alpha \neq 0^{\circ}$ ) will not be discussed. The potential of propeller phase control as a means of noise reduction will also be given.

\section{IV.A. Rotation Rate and Rotation Direction}

The rotation rate has a large effect on the sound pressure levels given its direct relationship with tip Mach number. For the GL-10 blade geometry, tip Mach numbers over the RPM range of interest are between approximately $0.18-0.55$, corresponding to an excess of $30 \mathrm{~dB}$ difference in the maximum vehicle sound pressure level. Prior to studying the total vehicle noise, Fig. 8 gives the scaling of a single GL-10 propeller in forward flight with tip Mach number at an in-plane observer. The advance ratio and RPM are varied individually to span the GL-10 flight envelope. Knowing that thickness noise is maximum at this observer location, Fig. 8(a) shows that loading noise is still dominant. This fact holds true at all observers. Thickness noise at the blade passage frequency collapses very well with a power law based on tip Mach number, $M_{t}^{9.8}$. As expected, this trend is independent of advance ratio and flight speed. On the other hand, loading noise 
is less amenable to tip Mach number scaling. Various nondimensional parameters are attempted to better collapse the loading term. The parameter that worked best was an effective tip Mach number,

$$
M_{e f f}=\frac{M_{t}}{1+J\left(1-M_{t}\right)},
$$

as shown in Fig. 8(b). In this definition, the propeller advance ratio is used, but the form of this equation comes from Gopalan ${ }^{16}$ who identified (in terms of rotor advance ratio and the rotor advancing tip Mach number) this as an appropriate scaling for rotor thickness noise. Watts, Greenwood, \& Stephenson ${ }^{17}$ also found success when this scaling was applied to rotorcraft at different altitudes. The tonal loading first $\left(f_{0}\right)$ and second $\left(2 f_{0}\right)$ blade passage frequencies are now collapsed quite well. Such a result could assist in acoustically aware control strategies if computationally inexpensive noise predictions are needed. While the Mach number power is likely to change for a different blade geometry, performance versus noise decisions could be made by the vehicle in real-time. Of course, attached to this statement are the current assumptions which may or may not capture the dominant noise source.

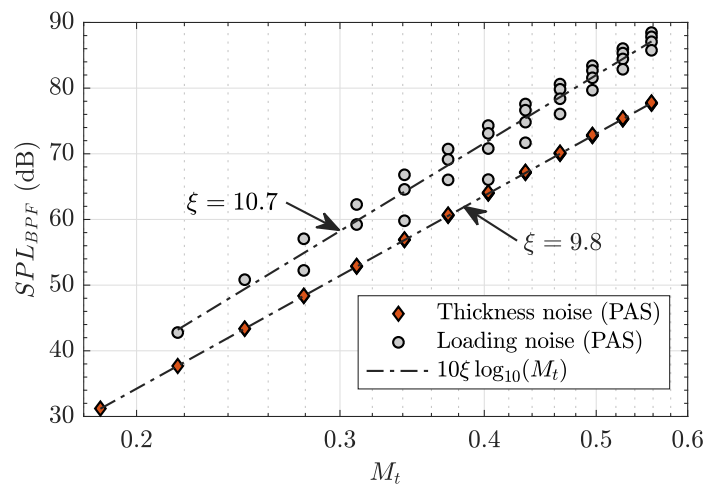

(a)

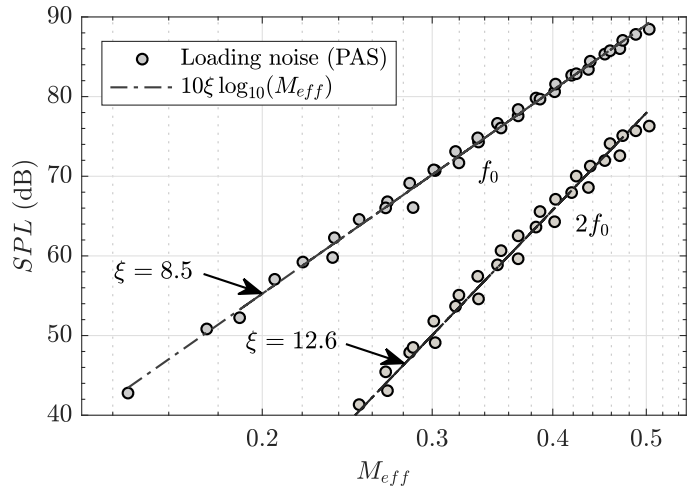

(b)

Figure 8. Sound pressure level scaling of an in-plane observer located at $(-12 R, 0,0)$ for (a) the blade passage frequency with tip Mach number, separated into thickness and loading. Scaling of the loading term at the blade passage and twice the blade passage frequency is given in (b) with an effective tip Mach number, $M_{e f f}$. All data are for the forward flight condition of a single isolated GL-10 propeller at $\alpha=0^{\circ}$.

The total noise from the set of ten propellers with the GL-10 layout will now be assessed. For this work, all propellers are set to the same RPM, i.e., spread frequency designs ${ }^{2}$ are not considered. Ground noise contours have similar dependence on the acoustic wavenumber as the bimonopole example in section III.B.1. Referring to Fig. 9a, lower rotation rates tend to produce a wider main lobe directly below the vehicle because the wingspan is comparable to the acoustic wavelength. Higher rotation rates increase the acoustic wavenumber, which in turn shrinks the width of the main lobe. Secondary sidelobes also begin to rival the main lobe at the highest rotation rate, again consistent with the bimonopole example. These elementary observations tend to hold when comparing noise contours in which all other parameters are fixed. However, there can be severe differences associated with the relative rotation directions amongst the set of propellers.

Rotation direction is an important parameter for aerodynamicists in terms of performance. Aerodynamic optimization of a vehicle can, however, depend on several other factors, opening the design space to other combinations of rotation direction. In an effort to act in prudence, several permutations are studied. Figure 9a contours are generated with all clockwise rotation $(+\Omega)$. During flight tests, the GL-10 had adjacent propellers rotating in opposite directions (Fig. 9b). Comparing these configurations, very similar contours are given. On the other hand, two additional sets of rotation directions give some interesting results. A significant increase in the width of the main lobe is produced when all propellers on the port side are rotating counterclockwise and the starboard are rotating clockwise (Fig. 9c). The opposite configuration (Fig. 9d) generates similar width in the energy distribution but is sliced into a set of three lobes. These two latter results (Figs. 9c-d) with ten propellers show very similar behavior compared to a set of two counter-rotating propellers (not shown). 

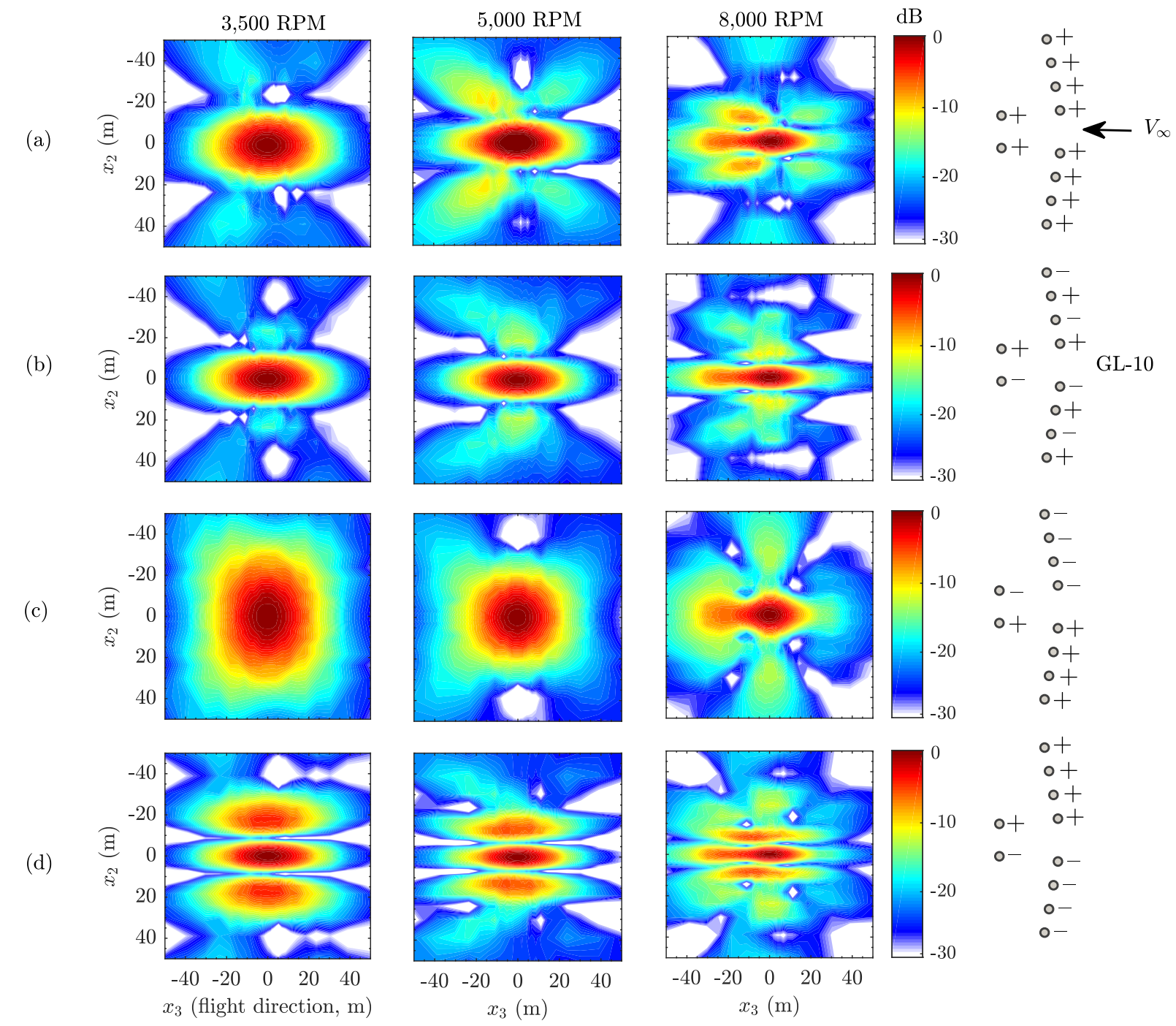

Figure 9. Noise contours displaying the effect of RPM and rotation direction for the GL-10. For all cases, $\alpha=0^{\circ}$, $\psi_{r_{1-10}}=0$ and $J=0.6$. Contours are normalized by the maximum level per case. 


\section{IV.B. Relative Phase}

Fixed relative phase (which implies equivalent rotation rates) between propellers was already shown in section III.B.1 to have the ability to affect noise patterns due to constructive and destructive interference. Referencing Fig. 10 for the phase definition, Fig. 11 gives examples of ground noise contours by modifying the relative phase of the GL-10. Clocking the phase by $\psi_{r}=60^{\circ}$ for all propellers on the starboard side (which is $N_{b} \psi_{r}=180^{\circ}$ of the blade passage frequency) shows cancellation along the $x_{2}=0$ line as the starboard side are all out of phase relative to the port side. The maximum sound pressure level can change significantly as certain phase combinations spread the acoustic energy more evenly over the ground plane. For instance, Figs. 11b-c show reduction in maximum SPL.

Current state-of-the-art UAVs are not outfitted with fixed relative blade positions or azimuthal position sensing/control hardware. In other words, the relative propeller phasing is random and unknown. This alone can make noise predictions very difficult, particularly as the number of individual propellers increases. The 5,000 RPM, $J=0.6$ case will be used to demonstrate this. When the relative phase is set at random to represent current GL-10 functionality, radiation patterns are highly variable due to coherent interference. Figure 12 illustrates this variability with three examples of random phase under the same flight conditions.

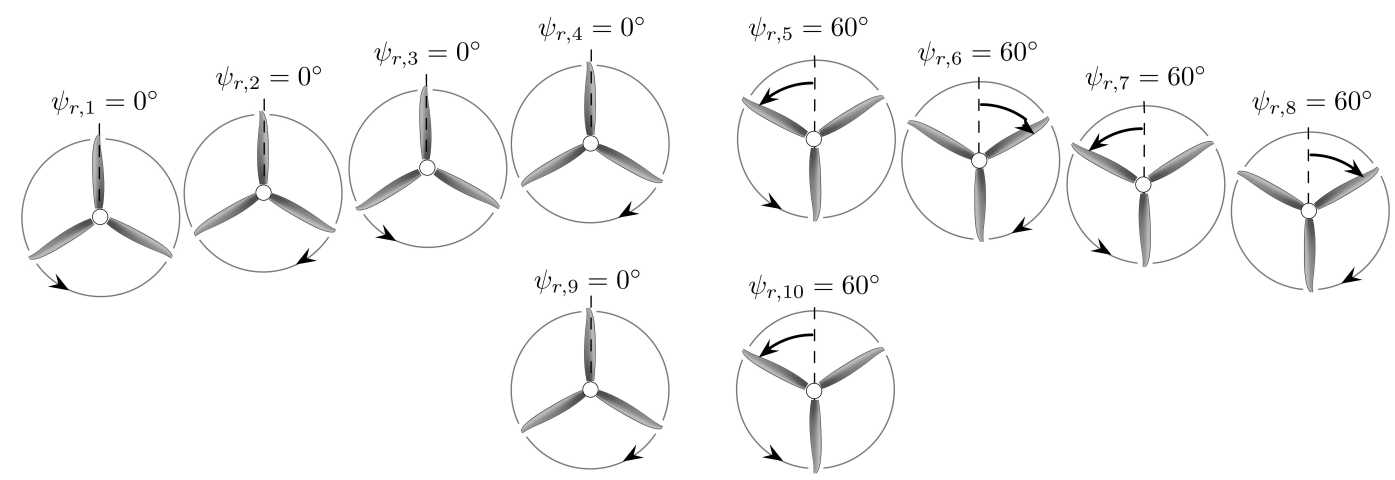

Figure 10. Example of relative phase offsets for the GL-10 as if viewing the vehicle from behind. The phase is defined in the direction of rotation, these corresponding to Fig. 11a.
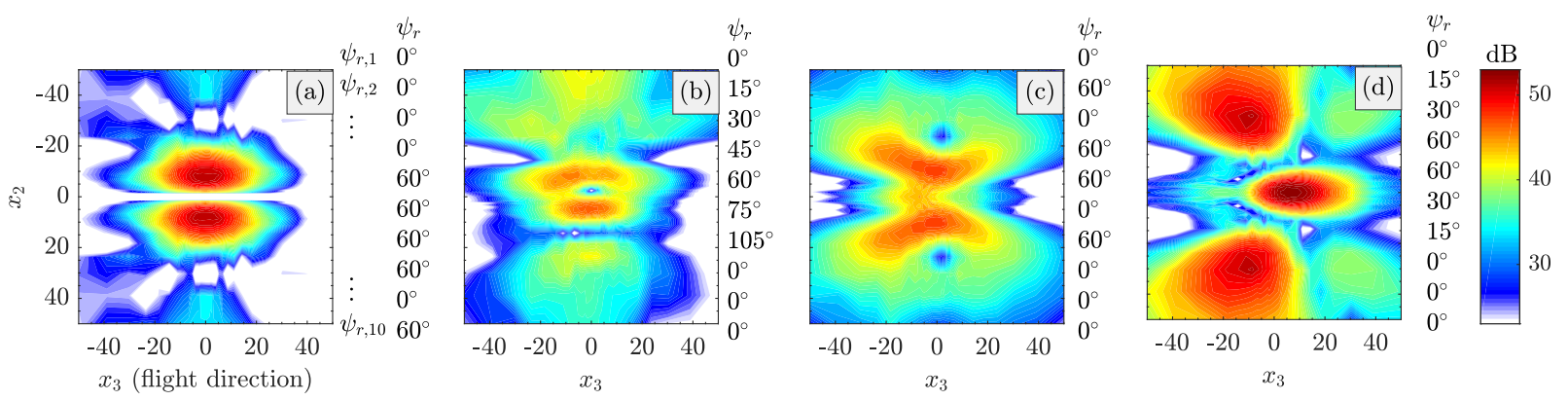

Figure 11. Difference in sound pressure on the ground plane with relative phase offset $\psi_{r}$ for the GL-10 at 5,000 RPM, $J=0.6$. The relative phases are listed to the right of each figure (refer to Fig. 10 for phase definitions).

Given these results, it is worthwhile to quantify the variation of noise levels with respect to relative phase. A Monte Carlo simulation is employed to determine confidence intervals of the ground contours. A total of 5,000 simulations are performed using uniformly distributed random phase for each propeller on the interval $[0,2 \pi)$ of the blade passage frequency. Statistics are well converged when using 5,000 instances. The cumulative probability distribution function $(\mathrm{CDF})$ of the $S P L$ is computed at each observer on the ground plane. The values at $P(0.025)$ and $P(0.975)$ on the distribution are evaluated, and their difference relative to the mean value determines the upper and lower $95 \%$ confidence interval bounds. Figure 13a gives the $\mathrm{CDF}$ and confidence intervals of a select observer. Note that attenuation due to observer distance does not affect the relative levels.

Confidence intervals are shown as a contour over all observers in Fig. 13b. Clearly, the confidence 

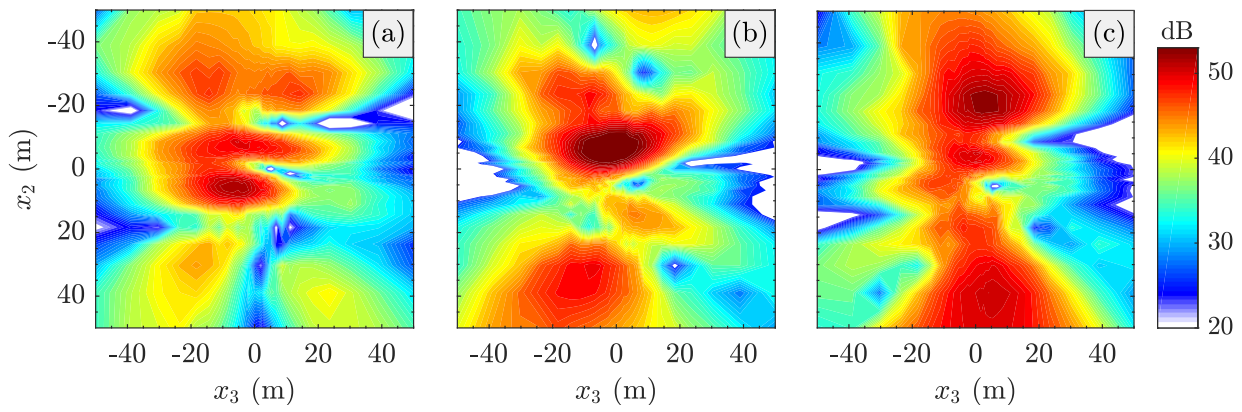

Figure 12. Three realizations of the sound pressure level on the ground plane when the relative phase is random for the GL-10 at 5,000 RPM, $J=0.6$. The relative rotation directions are specified in Fig. $9 \mathrm{~b}$.

interval is large everywhere spanning approximately $20-22 \mathrm{~dB}$. A major takeaway from this result is the large uncertainty in tonal levels of the GL-10 or similar distributed propulsion vehicles. However, this analysis represents an ideal case in which the blade passage frequency is identical for all propellers. In reality, motor controller error and wind gusts can introduce frequency or phase jitter, or alter the source or propagation characteristics such that the field deviates from a summation of coherent sources. This type of analysis would show that the confidence interval would be greatly reduced for a coherence less than unity but necessitates the need to properly model additional complex physics. Thus, the Monte Carlo analysis under ideal conditions likely represents the widest confidence interval in SPL.

The average effect of random phasing could be useful particularly when preparing noise regulations of DEP vehicles. Moreover, the average fields can serve as a reference case for section IV.C in which a phase control strategy is offered for noise reduction. Each observer location is averaged over the 5,000 random phase combinations. Figure 14 gives the stochastic directivity averages for three rotation rates. The maps show a great deal of similarity amongst each other, displaying maximum levels just aft of the in-plane angle. Also, the patterns parallel that of an isolated propeller (see $\alpha=0^{\circ}$ in Fig. 4). As the RPM is increased, the maximum SPL progresses more aft, which is expected as loading noise increases.

Due to the high variability in noise maps when relative phase is included, the metric chosen for the feasability study of Galles et al. ${ }^{3}$ is total radiated sound power, $P W L$, to serve as a placeholder until prediction capabilities mature. $P W L$ is a single-value measure of the source noise that is phase independent. Using ANOPP-PAS, the sound intensity is integrated over a full observer sphere surrounding a single isolated propeller. This value is then multiplied by the number of propellers, i.e., $P W L=10 \log _{10}\left(W_{s} / W_{0}\right)+$ $10 \log _{10}\left(N_{p}\right)$, in which $W_{s}$ is the power produced by a single propeller and $W_{0}=10^{-12}$ Watts. The values for three RPM conditions are given in Fig. 14.

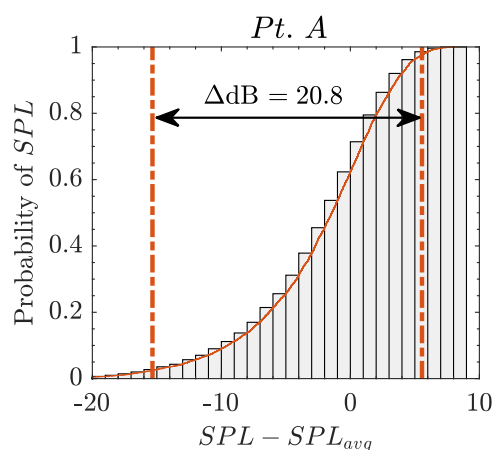

(a)

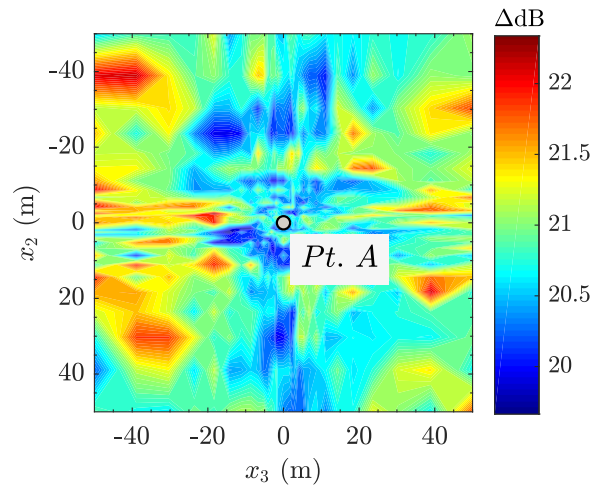

(b)

Figure 13. Demonstration of the $95 \%$ confidence interval calculation showing (a) histogram for select observer $(P t$. A). The contour in (b) summarizes the confidence interval for all observer locations on the ground plane for the 5,000 RPM and $J=0.6$ condition. The relative rotation directions are specified in Fig. $9 \mathrm{~b}$. 

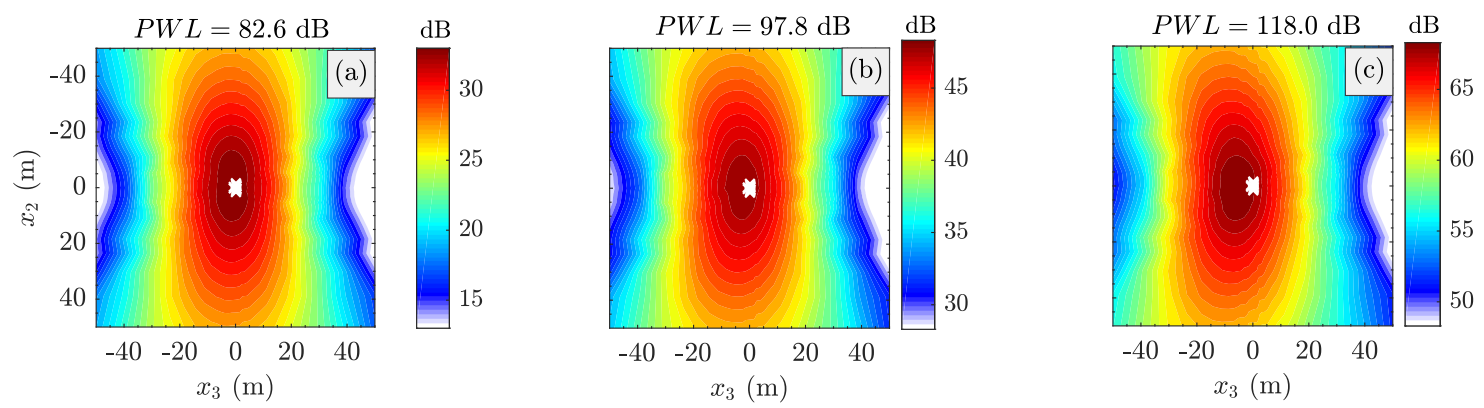

Figure 14. Stochastic averages of the blade passage frequency sound pressure level using 5,000 random relative phase combinations of the GL-10 for $J=0.6$ at (a) 3,500, (b) 5,000, and (c) 8,000 RPM. The relative rotation directions are specified in Fig. 9b. The phase-independent total radiated sound power metric, $P W L$, is also given for each case.

\section{IV.C. Phase Control Potential for Noise Reduction}

As just discussed, significant variations in noise can arise for random phase multipropeller vehicles operating at equivalent rotation rates. A reasonable follow up question is how can we leverage this variability to modify the directivity in a desirable manner? For example, if the vehicle is flying past a noise-sensitive area, can we push the acoustic energy in the other directions? A similar question has been well-studied in terms of reducing in-flight cabin noise (see, for example, Refs. 18-21). However, we are not aware of any analyses with the objective of reducing community noise. The large number of propellers typically found in DEP systems likely increase authority over directivity modification.

The number of propellers of the GL-10 makes it intractable to use equation 1 for all possible phase combinations. Johnston, Donham, \& Guinn, ${ }^{18}$ on the other hand, were able to compute all combinations for a four-propeller aircraft with $5^{\circ}$ azimuthal resolution to phase the propellers for cabin noise reduction. In general the number of possible combinations is $N_{c}=\left(2 \pi /\left(\Delta \psi_{r} N_{b}\right)\right)^{N_{p}-1}$, where $\Delta \psi_{r}$ is the azimuthal resolution in radians defined relative to the total azimuth. The exponent is one less than $N_{p}$, as one propeller is treated as the reference, e.g., $\psi_{r, 1}=0$. For the GL-10 with an azimuthal resolution of $\Delta \psi_{r}=0.0175 \mathrm{rad}$. $\left(1^{\circ}\right), N_{c}=\mathcal{O}\left(10^{18}\right)$. Reducing to a coarser resolution, $\Delta \psi_{r}=0.0873 \mathrm{rad}$. $\left(5^{\circ}\right), N_{c}=\mathcal{O}\left(10^{12}\right)$, still a very large value. Making matters worse, this calculation must be performed for every flight condition and noise reduction region of interest. To limit computational expense and following work of the antenna community (see, for example, Ares et al. ${ }^{22}$ and Boeringer \& Werner ${ }^{23}$ ), we turn to optimization techniques to solve for the phase angles.

Several optimization algorithms were attempted. As a first pass, Matlab's $f$ mincon ${ }^{24}$ function proved quick and provided a reasonable answer. However, fmincon is highly dependent on the initial values. In other words, the optimization has a tendency to get stuck in local minima. Particle swarm optimization (PSO) performed better in this regard. The major drawback of PSO is that tuning parameters need to be adjusted for largely differing propeller layouts, making this approach less attractive for determining the benefits of one configuration over another. For these reasons, a third technique was used; the multistart ${ }^{24}$ implementation of fmincon worked best for our purposes. Essentially, the optimization is run for 200 different initial value sets. Reasonable trends across different cases gave confidence a global minimum (or at least a local minimum close to the value of the global minimum) was captured. A Sobol sequence ${ }^{25}$ is defined to randomly distribute the initial values efficiently over the phase space. The phase set that minimizes the objective function across the multiple runs is chosen.

Figure 15 gives a visual representation of the capabilities of directivity modification at the blade passage frequency with phase control for the GL-10. Note that the Doppler shift associated with forward flight was not accounted for. The objective function was specified as the minimum average sound pressure level over a subset of ground observers. To test if phase control has enough authority to 'steer' acoustic energy away from a given direction, an octant of observers on the ground plane defines a given subset. As shown, phasing can effectively drop the levels by approximately $20 \mathrm{~dB}$ relative to the stochastic case in Fig. 14b over a given octant under these ideal conditions.

It is likely vehicle configurations other than the GL-10 may be even better suited for phase control. Current efforts are underway to identify vehicle properties, e.g., propeller spacing, rotation rates, and rotation directions, that allow phase control to be most effective. For example, Fig. 16 is the result of a canonical 
two by two propeller array with one diameter spacing in forward flight. Rotation is set to be all in the same direction. Similar reductions are found for each octant but with a less complicated lobe structure. Moreover, fore/aft symmetry of the propeller layout increases uniformity of phase control over all observer angles.
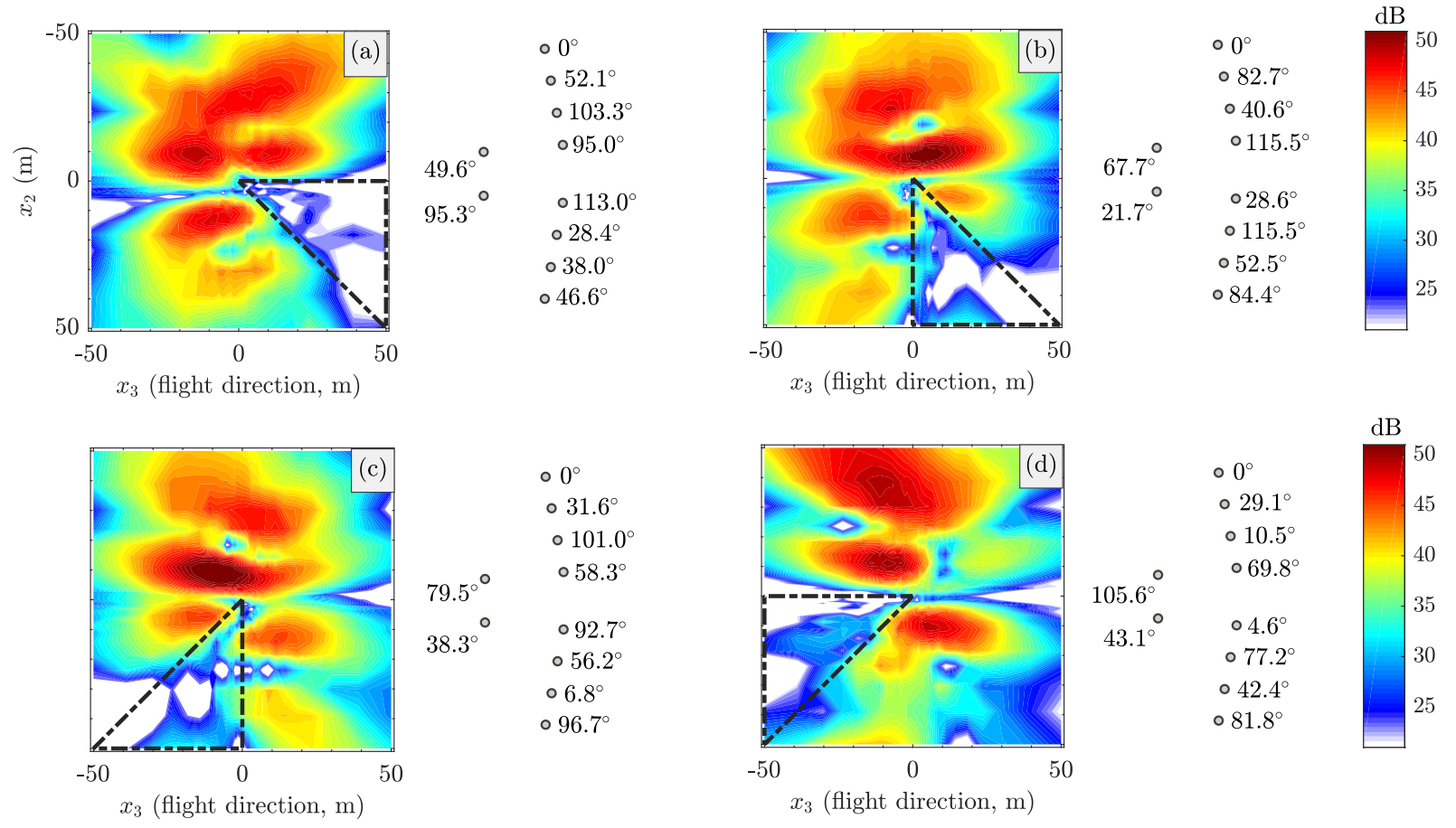

Figure 15. Example of directivity modification of the blade passage frequency on the ground plane via relative propeller phase control, for the GL-10 layout at 5,000 RPM, $J=0.6, \alpha=0^{\circ}$. The outlined regions are the observers given to the optimization for noise minimization, and the relative phase, $\psi_{r}$, of each propeller is given in degrees in the direction of rotation.

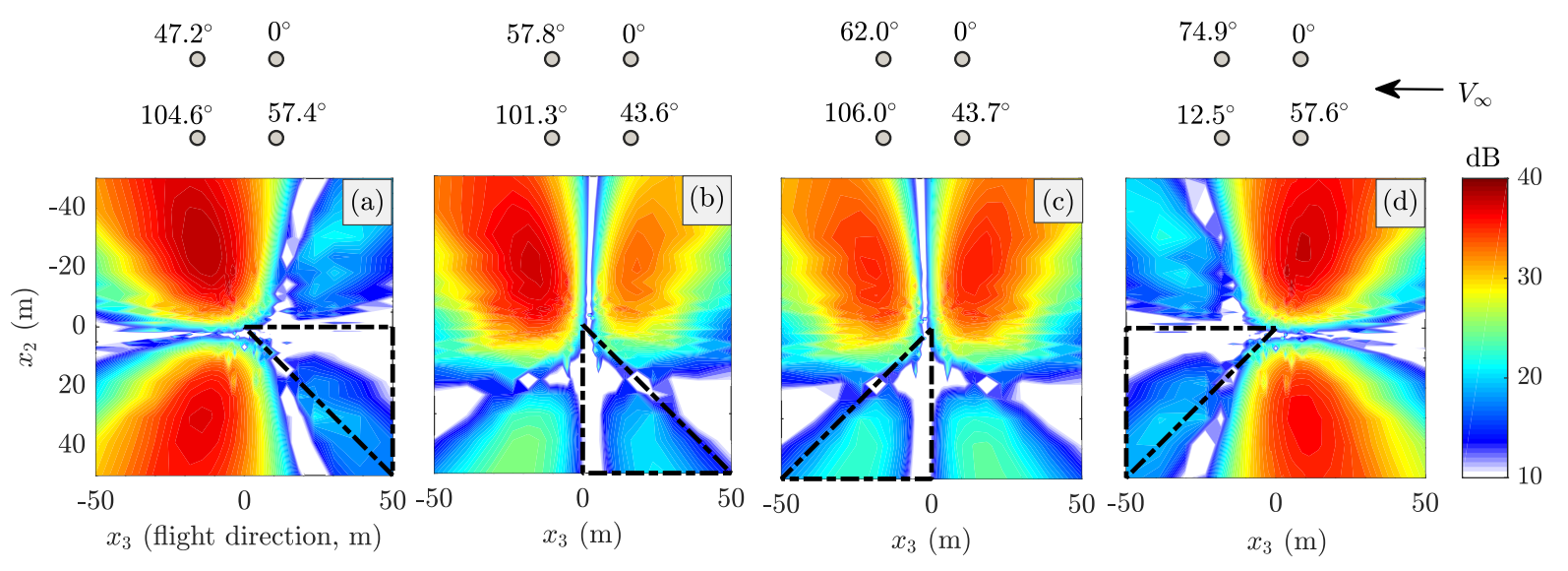

Figure 16. Example of directivity modification of the blade passage frequency on the ground plane via relative propeller phase control at 5,000 RPM, $J=0.6, \alpha=0^{\circ}$. The vehicle configuration is a two by two propeller array with hub spacing of a diameter. The outlined regions are the observers given to the optimization for noise minimization, and the relative phase, $\psi_{r}$, of each propeller is given in degrees. All propellers are rotating clockwise.

Until now, the rotation rates have been assumed constant without any deviation from the set phase value. This is an ideal case resulting in perfectly coherent signals between propellers. In actuality, the phase controller will have to continuously compensate for environment interaction such as wind gusts, turbulence ingestion, etc. Rizzi et al. ${ }^{2}$ studied the effect of a motor control error for a spread frequency design. The derivation equivalently applies to modeling a phase error (a direct result of a change in RPM) and is used 
here. Implementing, equation 2 now includes an additional term to modulate the phase argument,

$$
\Psi=2 \pi n_{h} f_{0, n} t+\tilde{\psi}_{m, n_{h}, n}+k_{n_{h}}\left(r_{m, n}-R_{s}\right)+N_{b} n_{h} \psi_{r_{n}}+2 \pi n_{h} N_{b} \epsilon \cos \left(2 \pi f_{\text {mod }} t+\phi_{e, n}\right),
$$

in which $\epsilon$ is the motor error that is assumed to be constant over all propellers given that the same hardware/control algorithm would likely be used. Note that $\epsilon$ is defined over the full propeller azimuth, e.g., $\epsilon=0.01$ is equivalent to $1 \%$ or a 3.6 degree depth of modulation to model the error. Additionally, $f_{\text {mod }}=5$ $\mathrm{Hz}$ is the frequency of modulation. The value is not particularly important when surveying mean-square pressures, so long as a full period of $1 / f_{\text {mod }}$ is modeled. $\phi_{e, n}$ is needed to model independent control of each propeller. That is, the phase error among propellers is not synced. Not including $\phi_{e}$ would lock the phase error of all propellers and virtually increase the coherence between the acoustic signals across the propeller array.

Figure 17 gives the optimized relative phase for the two by two propeller array. As expected, increasing the motor control error degrades the noise reduction potential. $0.1 \%$ error displays virtually no change relative to the case without error. Acoustic energy begins to be leaked into the region of interest when increasing the error to $1 \%$. By the time the error reaches $5 \%$, nearly all benefit is lost. Errors are not expected to exceed $1 \%$, however, based on current technology. Thus, phase control shows merit as a possible means of noise reduction technology. Additional considerations such as deviation from perfect coherence due to propeller spacing, turbulence, and installation effects also need to be investigated. For instance, wind gusts would likely generate short bursts of high acoustic levels. But, integrated time metrics such as sound exposure level will likely be reduced in a significant manner in the optimized direction.
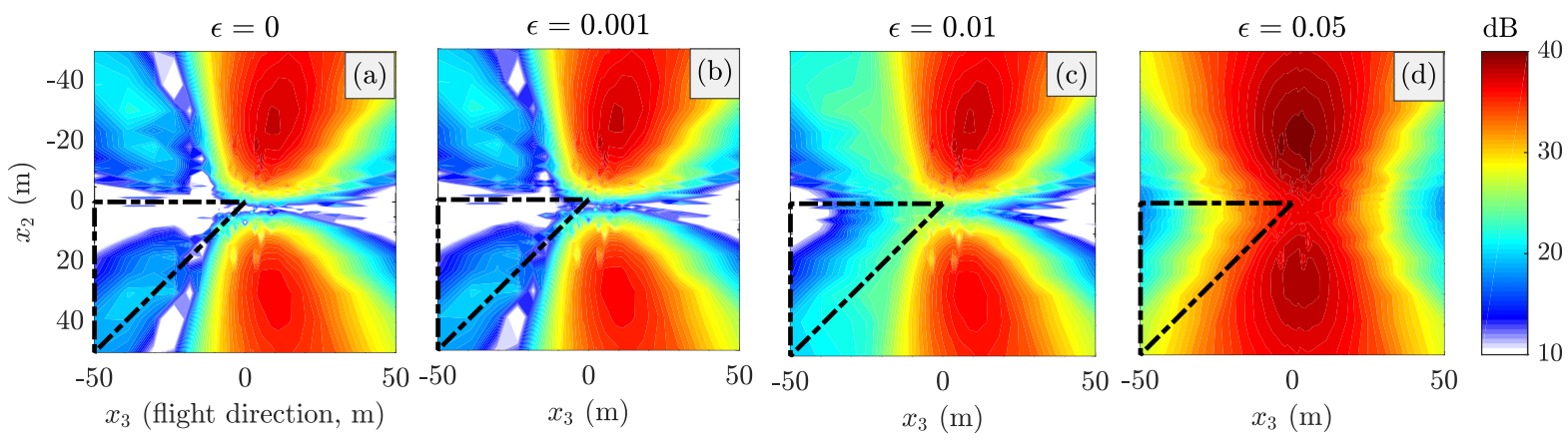

Figure 17. Notional effect of motor control error $(\epsilon)$ using equation 5 as the argument of equation 1 for the same canonical case in Fig. 16d.

\section{Extension to Vertical Flight Mode}

Until this point, only the forward flight mode has been discussed. Given that the GL-10 and many other UAVs have VTOL capabilities, it is worthwhile to incorporate vertical flight in the acoustic prediction. For this, PAS will also be used but with a few modifications. First, the coordinate system is rotated $90^{\circ}$ such that observer coordinates from the forward flight mode now correspond to conditions of a rotor in vertical flight (note that 'rotor' and 'propeller' will be used to describe the condition in which the axis of rotation is perpendicular or parallel to the ground plane, respectively). Also, PAS requires a nonzero freestream velocity as its intended use is for propellers in forward flight. Zawodny, Boyd, \& Burley ${ }^{26}$ found that in addition to the coordinate change, imposing a small nonzero freestream velocity manages to reasonably capture experimental trends of a rotor in hover.

While comparisons with experiments have been well documented for propellers, the accuracy of PAS in a rotor configuration has just recently been assessed. Experiments were conducted for the GL-10 propeller geometry (see Ref. 26 for details on a very similar setup), and were performed in the anechoic Structural Acoustics Loads and Transmission facility ${ }^{27}$ (SALT) under static conditions. Raw data are only kept prior to the effects of recirculation in the anechoic room. Hence, ingestion of nonuniform inflow that would otherwise greatly increase harmonic levels is not considered. Figure 18 shows good agreement between PAS and directivity of an isolated rotor at the blade passage frequency.

Figure 19 gives the predicted ground noise contours for the GL-10 in a vertical flight mode. Other than the change in propeller orientation and flight speed (now set to a near-hover condition), the same operating 
conditions as Fig. 9b are set. As expected, similar levels of steady loading and thickness noise relative to the forward flight condition are produced for the various RPM settings. Directly below the vehicle now becomes the location where the minimum noise is observed as it corresponds nearly to the rotor hub axes. Thickness and loading are both pushed out radially on the observer plane.

Phase control can also be applied to the vertical flight mode. Authority in the ability to steer the rotor noise is again displayed for the GL-10 in Fig. 20. Caution should be used when studying these results. Unsteady loading due to each blade chopping through the wake of its predecessor can drastically change the dominant source mechanisms. In other words, under low vertical speeds (near a hover state and particularly during vertical landing), unsteady loading noise, which is not captured in the present analysis, is likely to dominate the overall vehicle signature.
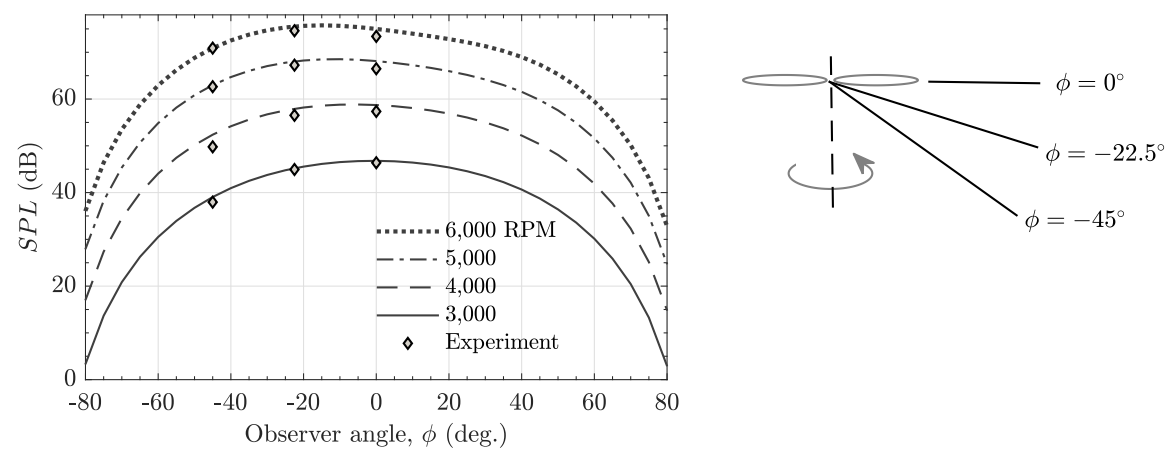

Figure 18. Comparison of PAS prediction and experimental measurements of a single GL-10 rotor in hover mode.
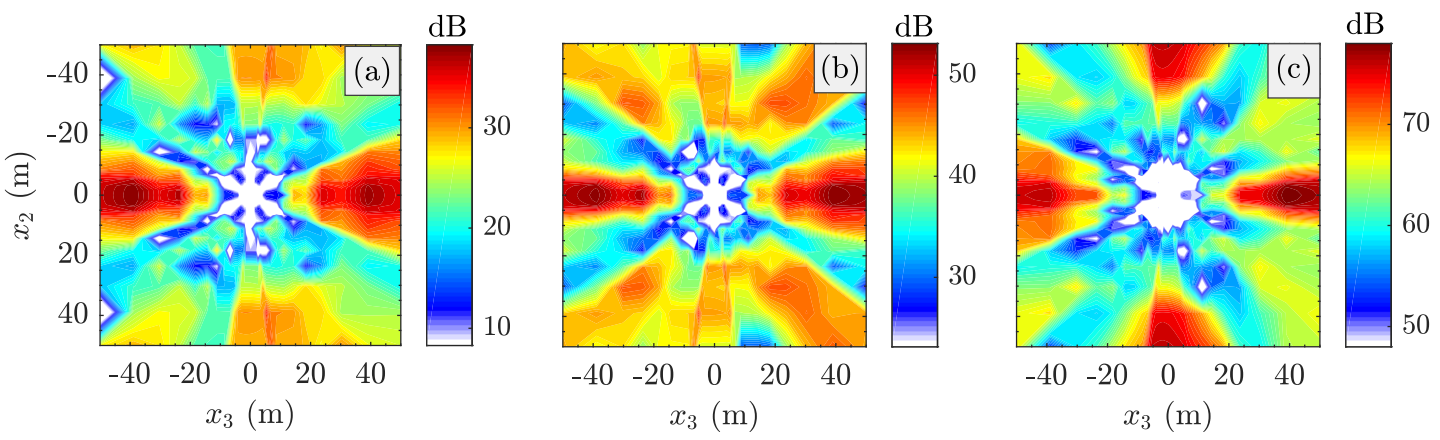

Figure 19. Ground plane noise contours for the GL-10 in a near hover mode at (a) 3,500, (b) 5,000, and (c) 8,000 RPM. The relative rotor phases are $\psi_{r, 1-10}=0$.
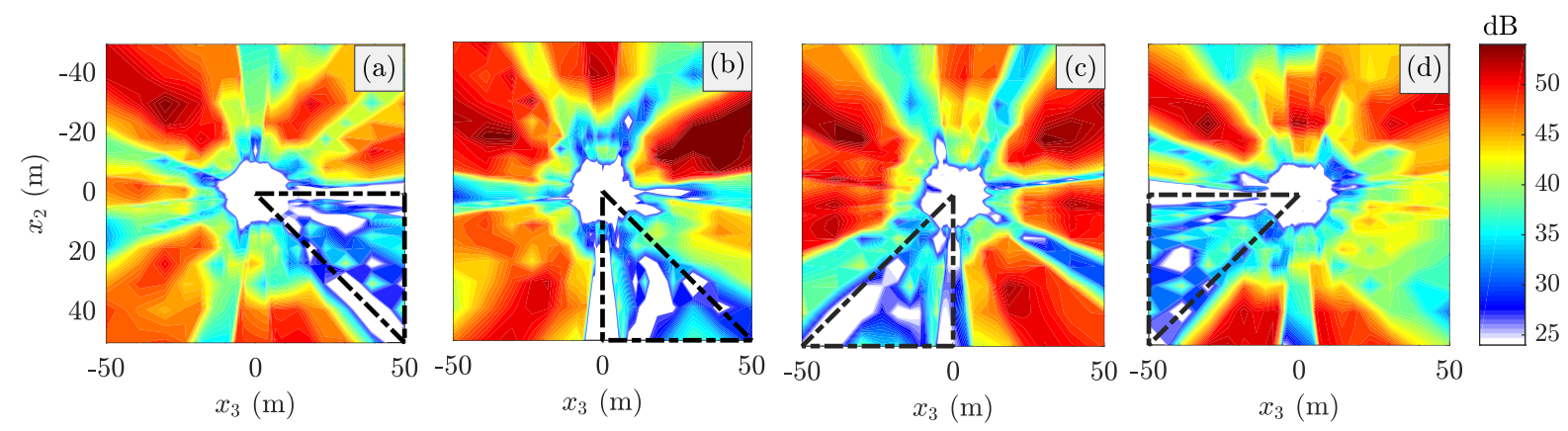

Figure 20. Example of directivity modification of the blade passage frequency on the ground plane via relative propeller phase control, for the GL-10 layout at 5,000 RPM in the vertical flight mode. The outlined regions are the observers given to the optimization for noise minimization. 


\section{Concluding Remarks}

A noise prediction scheme is introduced in an effort to generate an acoustic model that can be used for acoustically aware technology, that is, intelligent decision making by the flight control system for low-noise operation. Single propeller predictions show reasonable scaling with a Mach number based on blade motion. Total propulsive noise is estimated at far field observers by summing single propeller predictions.

Noise levels are most sensitive to RPM, but show significant directivity variability with relative rotation direction and propeller phasing, particularly when all propellers are rotating at the same rate. Monte Carlo simulations of relative phase combinations find confidence intervals spanning approximately $20 \mathrm{~dB}$ for any given observer. However, the average directivity patterns are qualitatively very similar to that of an isolated propeller.

Phase control, i.e., adjusting the relative azimuthal blade positions amongst the propulsors, suggests noise can be effectively steered away from a subset of observers, yielding a $20 \mathrm{~dB}$ reduction in the average sound pressure level across observers spanning 45 degrees. While several realistic effects will likely reduce this potential benefit, current capabilities of motor controllers are expected to be within the accuracy needed for phase control.

Finally, predictions and phase control of the vertical flight mode display similar maximum levels as the forward flight mode, but with a different directivity character. A small but nonzero flight speed is specified to avoid conditions where the sources modeled here are overcome by unsteady loading noise via self-wake ingestion.

\section{Acknowledgments}

This work was supported by the NASA Langley Lean Forward Autonomy Self Aware Vehicle project, and the NASA Aeronautics Research Mission Directorate, Revolutionary Vertical Lift Technology project. The authors wish to thank Mr. Matthew Galles and Dr. Noah Schiller of the NASA Langley Structural Acoustics Branch for numerous insightful discussions. Also, thanks to Dr. Irene Gregory, Mr. Kasey Ackerman, and Dr. John Cooper of the NASA Langley Dynamic Systems and Control Branch for their guidance on the GL-10 aircraft and its control system. Finally, thanks is due to Dr. Nikolas Zawodny of the NASA Langley Aeroacoustics Branch for his expert opinions and assistance with PAS-ANOPP and the experimental data in Fig. 18.

\section{References}

1 Dudley, M., "Enabling New Flight Concepts Through Novel Propulsion and Energy Architectures," Second Annual Transformative Vertical Flight Concepts Workshop, 2016.

2 Rizzi, S. A., Palumbo, D. L., Rathsam, J., Christian, A., and Rafaelof, M., "Annoyance to Noise Produced by a Distributed Electric Propulsion High-Lift System," 23rd AIAA/CEAS Aeroacoustics Conference, AIAA Paper 2017-4050, 2017.

3 Galles, M., Schiller, N., Ackerman, K., and Newman, B., "Feedback Control of Flight Speed to Reduce Unmanned Aerial System Noise," 24th AIAA/CEAS Aeroacoustics Conference, 2018.

4 Fredericks, W. J., Moore, M. D., and Busan, R. C., "Benefits of Hybrid-Electric Propulsion to Achieve 4X Increase in Cruise Efficiency for a VTOL Aircraft," AIAA Aviation Technology, Integration, and Operations (ATIO) Conference, AIAA Paper 2013-4324, 2013.

5 Brooks, T. F., Pope, D. S., and Marcolini, M. A., "Airfoil Self-Noise and Prediction," NASA RP-1218, 1989.

6 Magliozzi, B., Hanson, D., and Amiet, R., "Aeroacoustics of Flight Vehicles: Theory and Practice. Volume 1: Noise Sources," NASA TR 90-3052, 1991.

7 Huff, D. L., Henderson, B. S., and Envia, E., "Motor Noise for Electric Powered Aircraft," 22nd AIAA/CEAS Aeroacoustics Conference, AIAA Paper 2016-2882, 2016.

8 Rothhaar, P. M., Murphy, P. C., Bacon, B. J., Gregory, I. M., Grauer, J. A., Busan, R. C., and Croom, M. A., "NASA Langley Distributed Propulsion VTOL Tilt-Wing Aircraft Testing, Modeling, Simulation, Control, and Flight Test Development," 14th AIAA Aviation Technology, Integration, and Operations Conference, AIAA Paper 2014-2999, 2014. 
9 Wald, Q. R., "The aerodynamics of propellers," Progress in Aerospace Sciences, Vol. 42, No. 2, 2006, pp. 85-128.

10 Nguygen, L. C. and Kelly, J. J., "A Users Guide for the NASA ANOPP Propeller Analysis System," NASA CR-4768, 1997.

11 Zorumski, W. E., “Aircraft Noise Prediction Program Theoretical Manual, Part 1,” NASA TM-93199, 1982.

12 Schlichting, H., Boundary-Layer Theory, McGraw-Hill, 1979.

13 Farassat, F., "Theory of Noise Generation from Moving Bodies with an Application to Helicopter Rotors," NASA TR-R-451, 1975.

14 Padula, S. and Block, P., "Predicted changes in advanced turboprop noise with shaft angle of attack," Journal of Propulsion and Power, Vol. 1, No. 5, 1985, pp. 381-387.

15 Blackstock, D. T., Fundamentals of Physical Acoustics, John Wiley \& Sons, 2000.

16 Gopalan, G. and Schmitz, F., "Far-field near-in-plane harmonic main rotor helicopter impulsive noise reduction possibilities," American Helicopter Society 64th Annual Forum, 2008.

17 Watts, M. E., Greenwood, E., and Stephenson, J., "Measurement and Characterization of Helicopter Noise at Different Altitudes," American Helicopter Society 72nd Annual Forum, 2016.

18 Johnston, J., Donham, R., and Guinn, W., "Propeller signatures and their use," Journal of Aircraft, Vol. 18, 1981, pp. 934-942.

19 Magliozzi, B., "Synchrophasing for Cabin Noise Reduction of Propeller-Driven Airplanes," 8th AIAA Aeroacoustics Conference, AIAA Paper 1983-0717, 1983.

20 Fuller, C., "Analytical model for investigation of interior noise characteristics in aircraft with multiple propellers including synchrophasing," Journal of Sound and Vibration, Vol. 109, No. 1, 1986, pp. 141-156.

21 Huang, X., Wang, Y., and Sheng, L., "Synchrophasing control in a multi-propeller driven aircraft," Journal of Engineering for Gas Turbines and Power, Vol. 136, No. 11, 2014.

22 Ares-Pena, F. J., Rodriguez-Gonzalez, J. A., Villanueva-Lopez, E., and Rengarajan, S., "Genetic algorithms in the design and optimization of antenna array patterns," IEEE Transactions on Antennas and Propagation, Vol. 47, No. 3, 1999, pp. 506-510.

23 Boeringer, D. W. and Werner, D. H., "Particle swarm optimization versus genetic algorithms for phased array synthesis," IEEE Transactions on antennas and propagation, Vol. 52, No. 3, 2004, pp. 771-779.

24 The Mathworks, Inc., Natick, Massachusetts, MATLAB Optimization Toolbox, version 9.3.0.713579 (R2017b), 2017.

25 Greenwood, E., Fundamental Rotorcraft Acoustic Modeling from Experiments (FRAME), Ph.D. Dissertation, University of Maryland, 2010.

26 Zawodny, N., Boyd, D., and Burley, C., "Acoustic Characterization and Prediction of Representative, Small-Scale Rotary-Wing Unmanned Aircraft System Components," 72nd American Helicopter Society (AHS) Annual Forum, 2016.

27 Grosveld, F. W., "Calibration of the structural acoustics loads and transmission facility at NASA Langley Research Center," INTER-NOISE and NOISE-CON Congress and Conference Proceedings, Vol. 1999, Institute of Noise Control Engineering, 1999, pp. 1541-1546. 